\title{
Nanoassembled Thin-film-coated Quartz Crystal Microbalance Odor Sensors for Environmental and Human Breath Ammonia Assessments
}

\author{
Seung-Woo Lee, ${ }^{1,4^{*}}$ Roman Selyanchyn, ${ }^{1}$ Shunichi Wakamatsu, ${ }^{2}$ and Kenshi Hayashi ${ }^{3}$ \\ ${ }^{1}$ Graduate School of Environmental Engineering, University of Kitakyushu, \\ 1-1 Hibikino, Kitakyushu, Fukuoka 808-0135, Japan \\ ${ }^{2}$ Nihon Dempa Kogyo Co. Ltd., 1-3-1 Kashiwadai-minami, Chitose, Hokkaido 066-0009, Japan \\ ${ }^{3}$ Graduate School of Information Science and Electrical Engineering, Kyushu University, \\ 744 Motooka, Fukuoka 819-0395, Japan \\ ${ }^{4}$ EnH Japan Co. Ltd., Kitakyushu Science and Research Park, \\ 1-103 Hibikinokita, Kitakyushu, Fukuoka 808-0137, Japan
}

(Received September 1, 2017; accepted January 5, 2018)

Keywords: quartz crystal microbalance, ammonia, polyelectrolyte, layer-by-layer (LbL) deposition, odor sensor, breath monitoring

In this short review, we describe our recent approaches and strategies for the development of quartz crystal microbalance (QCM)-based odor sensor systems for environmental and human health monitoring. QCM electrodes, which were modified with sensitive nanometerthick coatings via layer-by-layer ( $\mathrm{LbL}$ ) deposition, were used to develop a system for measuring significant environmental changes in indoor air. Identification of the origin of environmental changes was possible via the differential signal analysis of obtained data. The sensors showed different responses to humidity changes, hazardous gas (ammonia), or cigarette smoke exposure. In addition, QCM sensors with porous films comprising silica nanoparticles and poly(allylamine hydrochloride) (PAH) were fabricated via an electrostatic self-assembly method and they exhibited considerable sensitivity to relative humidity (RH). The infusion of poly(acrylic acid) (PAA) into the multilayer porous film enabled the construction of a highly sensitive and selective QCM sensor device for the detection of gaseous ammonia. Two types of QCM sensors, with and without PAA, enabled the simultaneous quantitative detection of humidity and ammonia. Preliminary tests were conducted to detect low concentrations of ammonia in human breath, which are of clinical relevance. The results obtained showed that the sensor can detect ammonia in human breath at pathological levels (greater than $3 \mathrm{ppm}$ ).

\section{Introduction}

Odors are generally determined by the combination of volatile organic and inorganic compounds that have a high vapor pressure even at ordinary room temperature, and are identified and described by humans' natural sense of smell. Various living organisms, from

*Corresponding author: e-mail: leesw@kitakyu-u.ac.jp

http://dx.doi.org/10.18494/SAM.2018.1734 
simple bacteria to complex mammals (including humans), respond to environmental odors; these chemical signals play a major role in feeding, territorial recognition, sexual behavior, and detection of potentially harmful events and materials such as fire, gas, and rancid food. ${ }^{(1)}$ Therefore, the description of odors is of great analytical importance in several fields of human activity.

One of the recent trends in analytical sciences is the investigation of odors for medical diagnostics. ${ }^{(2)}$ Odorants vary widely in structure and include many chemical classes; however, humans have a limited capacity to identify individual odorants in mixtures. ${ }^{(3)}$ The development of sophisticated laboratory systems and small sensor devices capable of being integrated with personal and tablet computers and smartphones for odor analysis is underway to create novel diagnostic systems. The search for a correlation between odor signals and health conditions is expected to be the basis of future noninvasive disease diagnostics, self-assessment, or self-care and monitoring. ${ }^{(4)}$ A variety of odorous compounds are emitted from the human organism in sweat, urine, and breath. The assessment of odors using olfaction has a long history and is still used as a gold standard in some fields, such as in wine quality assessment. Olfaction, however, most often provides only qualitative detection; thus, a strong need exists for the development of objective instruments for the detection of compounds and concentrations that lie below the limit of human perception.

The quartz crystal microbalance (QCM) is a widely used mass-sensitive transducer owing to the simplicity of its operation. In most cases, the QCM oscillation frequency response to the mass loaded on the active electrode surface follows the Sauerbrey Eq. (1), ${ }^{(5)}$

$$
\Delta f=-\frac{2 f_{0}^{2}}{A \sqrt{\mu_{q} \rho_{q}}} \Delta m,
$$

where $f_{0}$ is the resonance frequency of the unloaded QCM, $A$ is the active area of the crystal, and $\mu_{q}$ and $\rho_{q}$ are the quartz shear modulus $\left(2.947 \times 10^{11} \mathrm{~g} \cdot \mathrm{cm}^{-1} \cdot \mathrm{s}^{-2}\right)$ and density $\left(2.648 \mathrm{~g} \cdot \mathrm{cm}^{-3}\right)$, respectively. QCM-based sensors have gained popularity in recent years and have been used in a variety of fields, ${ }^{(6,7)}$ including biotechnology, ${ }^{(8)}$ drug and surfactant research, ${ }^{(9,10)}$ biosensing, ${ }^{(11-15)}$ for gas sensing purposes, ${ }^{(16-18)}$ and as an element of electronic tongue or nose devices. ${ }^{(19,20)}$

Over the last several years, we have developed QCM-based gas sensors modified with functional nanothin films deposited via layer-by-layer $(\mathrm{LbL})$ deposition. In our initial approach to gas sensing, $\mathrm{TiO}_{2}$ ultrathin films that were alternately assembled with layers of poly(acrylic acid) (PAA) by the surface sol-gel process were used for ammonia detection. ${ }^{(17)}$ We have also reported the creation of reliable QCM humidity sensors modified with porphyrin-based nanothin films, ${ }^{(21)}$ which could also be applied to daily needs, for instance, the monitoring of indoor environmental quality. ${ }^{(22)}$ Recently, we have reported a respiration assessment system, based on QCM electrodes, which possessed a surface modified with nanoassembled thin films. ${ }^{(23,24)}$ We were able to obtain an online breathing record (respiratory pattern) owing to the quick response of the QCM sensors to the fast changes in relative humidity $(\mathrm{RH})$ in human breath samples. These changes depend on alterations in human breathing styles, such as in speed, intensity, and time of inhalation and exhalation. 
In this paper, we introduce our two case studies for the development of QCM-based odor sensor systems for real environment measurements from several sources (humidity, ammonia gas, or cigarette smoke) ${ }^{(22)}$ and for monitoring human breath, ${ }^{(25)}$ particularly breath ammonia. The proposed system, with all the necessary built-in units, can be used to access mechanical parameters and the chemical composition of environmental and human samples.

\section{Real Environment Measurements}

From the recent findings on indoor air quality (IAQ), it is evident that indoor air is often more polluted than outdoor air. ${ }^{(26)}$ This is mainly caused by insufficient ventilation, and therefore polluted indoor air is often a great health hazard. ${ }^{(27)}$ The creation of sensors for IAQ in buildings, which can monitor common parameters (such as temperature or humidity) and additionally certain chemical factors, is an important task for sensor technology development. Devices that can detect emergency conditions (e.g., fire occurrence and toxic chemical emission) and automatic ventilation systems to maintain IAQ are of great importance. Since conventional sensors that are currently used for chemical detection are usually highly influenced by humidity, the testing process for new sensors should account for operation under environment conditions that can often be harsh. ${ }^{(28)}$

For the development of a gas sensor system for real environment measurements from several sources (humidity, ammonia gas, or cigarette smoke), we examined the LbL deposition of porphyrin/polymer thin films on the surface of $9 \mathrm{MHz}$ QCM electrodes. ${ }^{(22)} \mathrm{RH}$ was selected because it is a highly important factor for human comfort and well-being inside buildings, ${ }^{(21)}$ ammonia was chosen to test the system as one of the most important analytes for IAQ assessment, ${ }^{(29)}$ and cigarette smoke was chosen because it is the simplest environmental factor that significantly influences IAQ. It is known that cigarette smoke can contain many

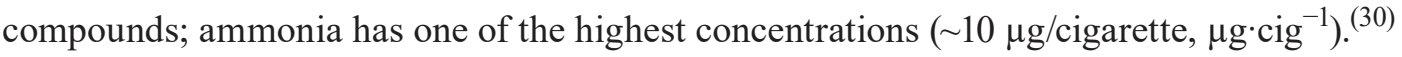

Figure 1 shows an example test confirming the reproducibility of QCM responses to both $100 \mathrm{ppm}$ ammonia exposures and humidity changes. These results were obtained using QCM electrodes with a 10-cycle LbL film that consists of a cationic polymer, poly(diallyldimethyl ammonium chloride) (PDDA; $M_{w}=200000-350000 \mathrm{~g} \cdot \mathrm{mol}^{-1}, 20 \mathrm{wt} \%$ in $\mathrm{H}_{2} \mathrm{O}$ ), and an anionic porphyrin, tetrakis-(4-sulfophenyl)porphine or tetrakis-(4-sulfophenyl)porphine manganese(III) chloride (TSPP or MnTSPP, respectively). To approximate environmental change, the raw signals obtained from the measuring system were differentiated. The differential approach makes "humidity-independent" ammonia events clearly distinguishable in the time-differential signal owing to several features: the four small negative pulses in Fig. 1(c) are not associated with any humidity change [Figs. 1(a) and 1(b)] and the response of the TSPP-modified electrode is higher owing to the slightly higher binding capacity of ammonia to TSPP than to MnTSPP [Fig. 1(c)]. This difference between the two films may be explained by the formation of a $J$-aggregate due to electrostatic interactions between the TSPP molecules that have protonated pyrrole rings and the deprotonated sulfonates in a molecule, ${ }^{(31)}$ whereas MnTSPP is present in the monomeric state. The $J$-aggregates of TSPP inside the film act as specific binding sites for ammonia. ${ }^{(32)}$ The average time-differential responses of the four pulses for the sudden appearance of 100 


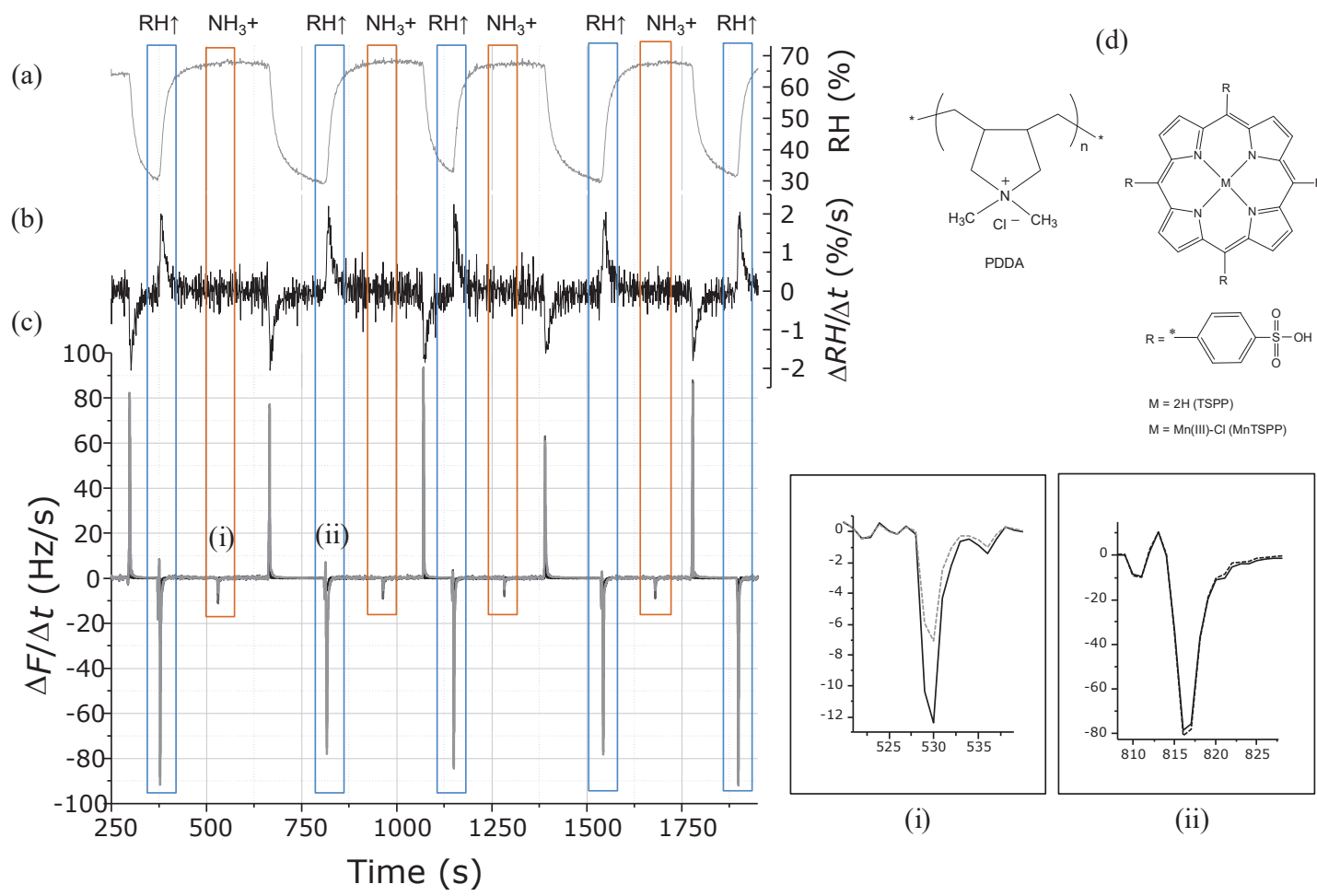

Fig. 1. (Color online) Comparison of dynamic QCM responses for repeated exposure to humidified air and ammonia in humidified air (ca. 68\% RH). (a) Raw RH changes. (b) Time-differential humidity changes. (c) Time-differential QCM signals for both QCM electrodes (light grey: PDDA/MnTSPP; black: PDDA/TSPP). The magnified insets show a characteristic response to (i) ammonia addition at high RH (the first one among four small negative peaks) and (ii) humidity increase from ca. 30 to $68 \%$ RH (the first one among five large negative peaks). The high positive peaks are responses to flushing the system with dry air. (d) Molecular structures of PDDA and porphyrins (TSPP and MnTSPP) used for film preparation.

ppm $\mathrm{NH}_{3}$ gas in the atmosphere were estimated to be $11.5 \pm 2.5$ and $6.7 \pm 1.3 \mathrm{~Hz}$ for the PDDA/ TSPP and PDDA/MnTSPP films, respectively; however, these values are quite small compared with the responses to humidity. Nevertheless, the system recognizes when a gaseous influence is present in a stable high-humidity environment, indicating a reproducible response to a sudden increase in ammonia gas concentration in the environment.

Figure 2 shows a comparison of the raw signal $\Delta F(t)$, the time-differentiated signal $\Delta F / \Delta t(t)$, and the differentiated humidity change signal $\Delta R H / \Delta t(t)$, where $\Delta F / \Delta t(t)$ and $\Delta R H / \Delta t(t)$ describe the speed of the frequency and RH changes, respectively. The importance of the differentiated signal is that slow environmental changes are neglected, such as the baseline drift and slow humidity change due to air conditioning. However, significant and fast environmental alterations are clearly observed. As can be seen from Fig. 2, every odorous event that was performed in the investigation room at a temperature between $20-25^{\circ} \mathrm{C}$ and $\mathrm{RH}$ near $50 \%$ is reflected on the time-differential plot, $\Delta F / \Delta t(t)$. From the time-differential QCM signals, we can observe a clear difference between a fast humidity change (dashed line rectangles) and sudden ammonia or cigarette smoke exposure (dotted line rectangles); however, the conventional 


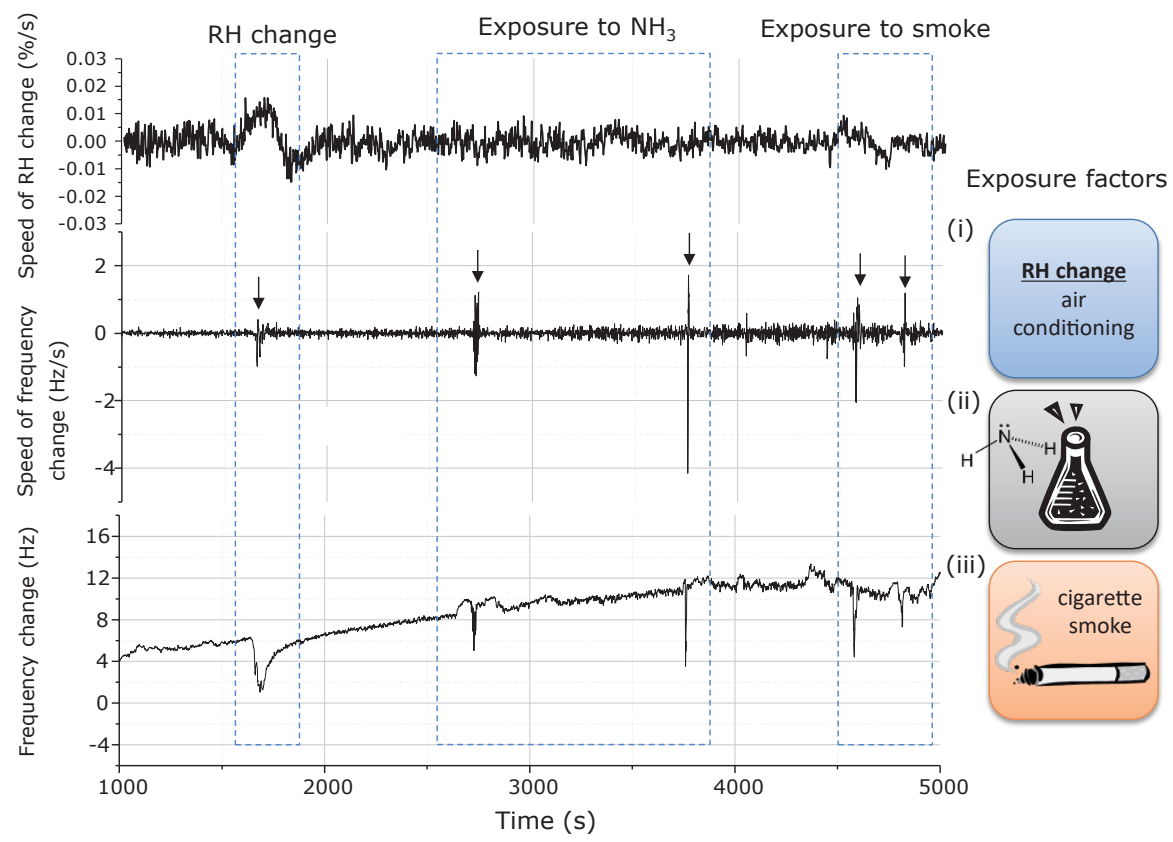

Fig. 2. (Color online) Comparison of QCM sensor response during exposure to different environmental gases showing differentiated signal. "Humidity-independent" odorous events are clearly distinguishable in the differentiated signal. A noise increase is observed when cigarette smoking is initiated.

humidity logger did not show changes as much as those observed by the QCM sensor when it was exposed to ammonia or cigarette smoke. The difference in the results is explained by the fast response of the QCM sensor; this is the basis on which an alarm system or ventilation switches can be created. Discrimination between different events, whether related or not to the chemicals, is possible in big volume spaces (rooms) owing to the fact that in big rooms, the RH does not change dramatically, and any changes are usually slow. These changes will cause only insignificant changes (small peaks) in the differentiated signal, and thus they can be easily filtered as false events. These results indicate that real environment monitoring devices can be implemented using humidity sensors based on surface-modified QCM platforms.

\section{Ammonia Detection in Human Breath}

Ammonia, an important analyte widely employed in the chemical industry, is also a potential disease biomarker. In the human body, ammonia mainly originates from protein metabolism, where it is converted into urea and excreted in urine. ${ }^{(33)}$ However, it is also normally present in low concentrations in blood, feces, exhaled breath, and skin emanations. ${ }^{(34)}$ Recently, numerous investigations to assess the ammonia emitted in human breath and to determine its usefulness as a disease biomarker for physical disorders such as renal insufficiency, ${ }^{(35)}$ hepatic dysfunction, ${ }^{(36)}$ Helicobacter pylori infection, ${ }^{(37)}$ or halitosis ${ }^{(38)}$ have been reported. Physiologically normal levels of ammonia present in human breath lie below approximately $1 \mathrm{ppm}$; therefore, the ammonia emitted by the human body cannot be detected using olfaction, except in cases of very 
severe disease conditions, where the ammonia concentration exceeds the human perception limit of 55 ppm. ${ }^{(39)}$

Numerous methods can be used for ammonia detection; however, bench-top systems are often required for low-level detection. PAA is well known as a sensitive receptor for ammonia detection. ${ }^{(40)}$ It is composed of repeating units of carboxyl groups [Fig. 3(a)], which determine the chemical and electrochemical properties of the material. In the solid state, the acid functional groups are capable of hydrogen bonding via the oxygen atom of the carbonyl moiety [Fig. 3(b)], and specific interactions with bases are expected as a consequence of acid-base reactions. Because of its chemical structure, the interaction of PAA with water vapor and basic ammonia changes the chemical and electrochemical properties of the polymer. In combination with appropriate transducers, these changes can be utilized in chemical sensors for humidity and ammonia gas detection, thus extending the scope of PAA applications beyond established industrial uses. ${ }^{(41)}$

In general, the porosity of sensor films is one of the major parameters for high-sensitivity sensor fabrication. ${ }^{(25)}$ Nanoparticles are good candidates for porous LbL film preparation. As schematically shown in Fig. 3(c), after the first deposition of a cationic polymer, poly(allylamine hydrochloride) (PAH; $M_{w}=58000 \mathrm{~g} \cdot \mathrm{mol}^{-1}$ ), washing with water, and drying under $\mathrm{N}_{2}$ gas, the QCM electrode could be porously modified by immersing it into a solution of negatively

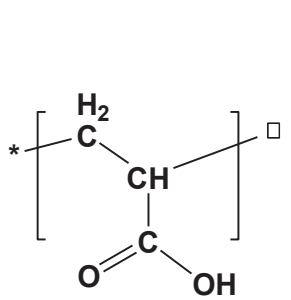

(a)

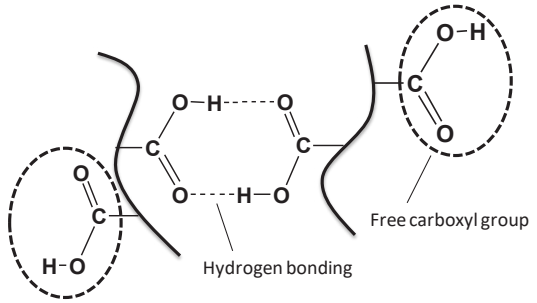

(b)

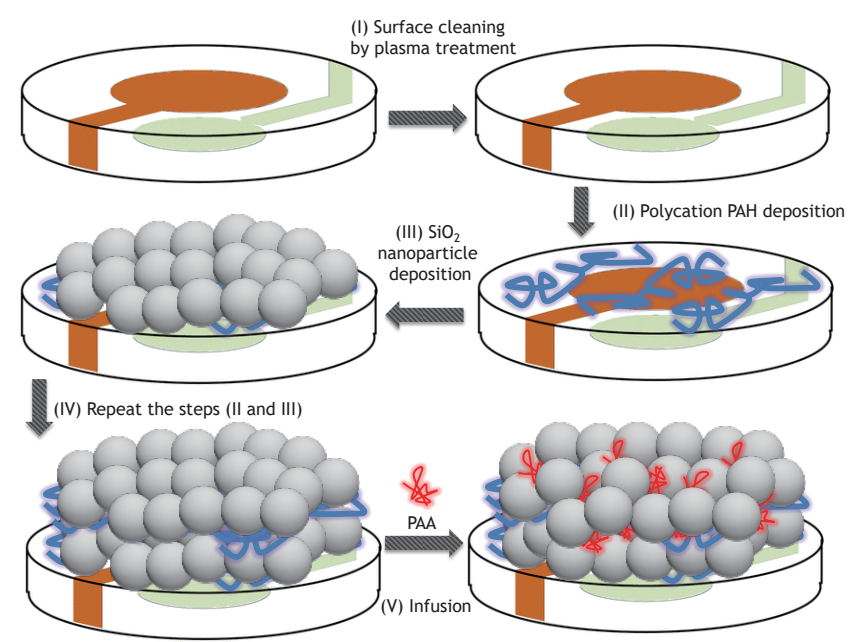

(c)

Fig. 3. (Color online) (a) Chemical structure of PAA and (b) PAA network and bonding possibilities. Schematic illustration of LbL film deposition using $\mathrm{PAH}$ and $\mathrm{SiO}_{2} \mathrm{NPs}$. 
charged $\mathrm{SiO}_{2}$ nanoparticles $\left(\mathrm{SiO}_{2} \mathrm{NPs}\right.$; particle size $\left.40-50 \mathrm{~nm}\right)$. The procedure was repeated until 5- and 10-cycle coatings (where one cycle is a $\mathrm{SiO}_{2} / \mathrm{PAH}$ bilayer) were prepared by the alternate adsorption of $\mathrm{PAH}$ and $\mathrm{SiO}_{2} \mathrm{NPs}$ onto the QCM. Then, the fabricated films were infused with PAA. After the infusion of PAA $(0.1 \mathrm{wt} \%)$ into the $\mathrm{SiO}_{2} / \mathrm{PAH}$ alternate film, the QCM electrode was rinsed with deionized water and dried using $\mathrm{N}_{2}$ gas. The self-assembly and infusion processes were monitored by measuring the QCM resonance frequency after every deposition cycle or infusion process. Figure 3(c) shows a schematic illustration of the LbL film deposition using PAH and $\mathrm{SiO}_{2} \mathrm{NPs}$.

Figure 4 shows a QCM sensor system (NAPiCOS, Nihon Dempa Kogyo Co., Ltd.) used for breath ammonia detection. Delivery of the sample and reference gases is achieved using a Sibata ${ }^{\circledR}$ minipump connected to the QCM chamber outlet. Temperature and humidity inside the chamber are recorded by additionally installed reference sensors, which are in a separate chamber in proximity to the QCM array. In addition, the chamber is designed to control the inside environment temperature in the range of 20 to $40{ }^{\circ} \mathrm{C}$. The temperature and $\mathrm{RH}$ sensors are the SII S-5814A (accuracy $\pm 2.5{ }^{\circ} \mathrm{C}$, working range -30 to $+100{ }^{\circ} \mathrm{C}$ ) and TDK CHS-UPS (accuracy $\pm 3 \%$, measurement range 5 to $95 \% \mathrm{RH}$ ), respectively. In addition, for cleaning purposes, the sensor system was flushed by air that was cleaned and dried through activated carbon and silica gel, respectively. Device operation was controlled by software (NAPiCOS 30A Real Time Monitor v.1.0).

Figure 5(a) compares frequency changes caused by the exposure of the QCM sensors with a coating of $\left(\mathrm{SiO}_{2} / \mathrm{PAH}\right)_{n}$ or $\left(\mathrm{SiO}_{2} / \mathrm{PAH}\right)_{n}+\mathrm{PAA}$ to water vapor and humidified ammonia gas at $35^{\circ} \mathrm{C}$, where the ammonia gas was obtained from a solution of $200 \mathrm{ppm}$ ammonia in water. After the humidity reached a certain level, humidified ammonia was added and a significant change in the PAA-modified QCM frequency was observed. As evident in the figure, the QCM sensors exhibited good repeatability of the sensor response to the same sample, except for the first run, which exhibited a slightly higher response. Such behavior of the sensors is probably related to the fact that adsorption at a small number of the binding sites is irreversible by simple air purging. However, all subsequent measurements are reversibly driven by the facile attachment of ammonia to the $\mathrm{COOH}$ functional groups of PAA by acid-base interactions.

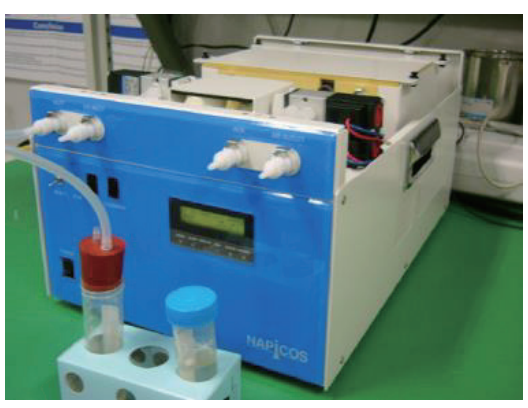

(a)

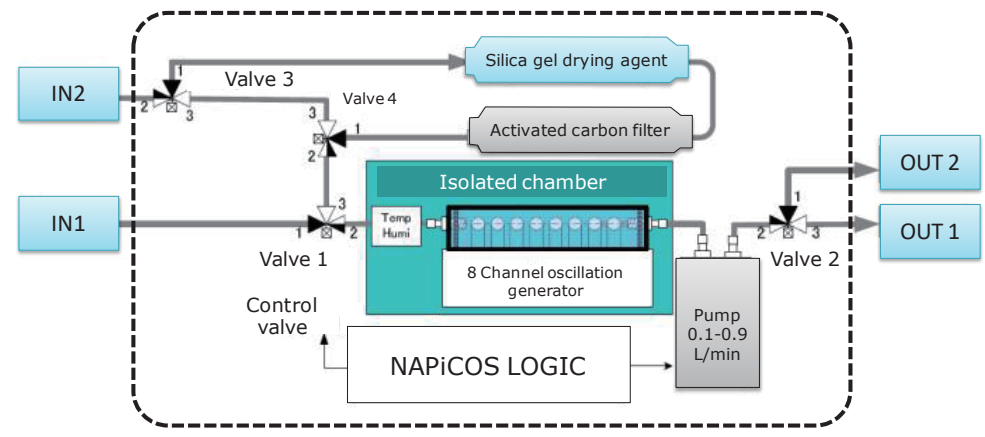

(b)

Fig. 4. (Color online) (a) Photograph of a prototype QCM sensor system and (b) arrangement of the elements inside the QCM sensor system used for breath ammonia detection. 


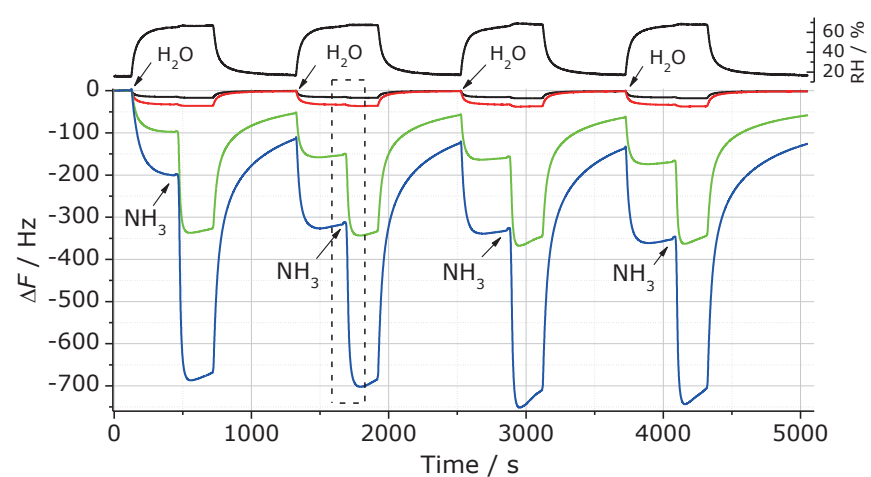

(a)

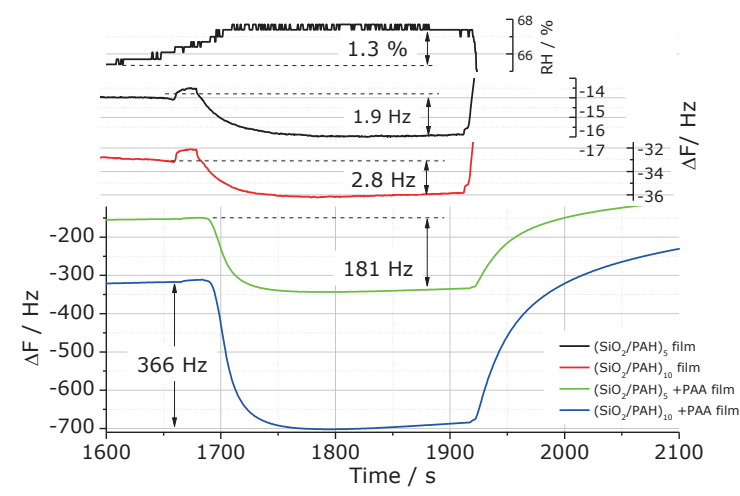

(b)

Fig. 5. (Color online) (a) Frequency changes induced by sequential exposure of QCM sensors to water vapor and $200 \mathrm{ppm}$ (sol) ammonia gas at $35^{\circ} \mathrm{C}$. (b) Detailed view of the area marked by the dashed rectangle in (a).

The absolute frequency change due to ammonia adsorption can be obtained by subtracting a response to humidity from that to humidified ammonia. The $\left(\mathrm{SiO}_{2} / \mathrm{PAH}\right)_{10}+\mathrm{PAA}-\mathrm{modified}$ QCM sensor shows a response of $389.1 \pm 14.8 \mathrm{~Hz}$ to the $200 \mathrm{ppm}$ (sol) ammonia gas at $35{ }^{\circ} \mathrm{C}$. Figure 5(b) shows a more detailed view of the frequency changes after the addition of ammonia in the second run of Fig. 5(a). Interestingly, the reference electrodes show very small responses to ammonia compared with those of the PAA-infused electrodes, which may have been caused by the small humidity increase (ca. 1.3\%) induced when the humidified ammonia sample was introduced.

All electrodes exhibited very stable and predictable behavior in all the experiments. The $\left(\mathrm{SiO}_{2} / \mathrm{PAH}\right)_{n}$ films exhibited a drift of approximately $2 \mathrm{~Hz}$ when measured for approximately 2 h under normal room conditions $\left(t \cong 25^{\circ} \mathrm{C}, \mathrm{RH} \cong 50 \%\right.$ ), whereas the PAA-infused electrodes exhibited an even smaller drift (under $1 \mathrm{~Hz}$ ). The limit of detection (LOD) was obtained from the linearity of the experimental data in the concentration range of 1-30 ppm ammonia and the data intercepted by the fitting line with a $3 \sigma$ level $(1.5 \mathrm{~Hz})$, where $\sigma$ was selected to be $0.5 \mathrm{~Hz}$ as a possible noise value. The LOD for ammonia in a humid atmosphere (at $35^{\circ} \mathrm{C}$ ) was estimated to be $2 \mathrm{ppm}$ (sol), corresponding to approximately $0.5 \mathrm{ppm}$ gas.

To investigate the possibility of ammonia detection in real samples, we proposed a function, $F^{W E}=f\left(F^{R E F}\right)$, where the working electrode (WE) and the reference electrode (REF) refer to the $\left(\mathrm{SiO}_{2} / \mathrm{PAH}\right)_{10}+\mathrm{PAA}$ and $\left(\mathrm{SiO}_{2} / \mathrm{PAH}\right)_{10}$ electrodes, respectively. When the $\left(\mathrm{SiO}_{2} / \mathrm{PAH}\right)_{10}$ electrode was used as a reference for the RH correlation, good linearity was observed $\left(r^{2} \rightarrow\right.$ 0.99), although a small degree of hysteresis was still observed. Figure 6 demonstrates how the function $\Delta F^{W E}=f\left(\Delta F^{R E F}\right)$ behaved during a 5-min test when $3 \mathrm{ppm}$ ammonia was sampled. Interestingly, when the samples with RH lower than the humidity level of the baseline were used, both electrodes exhibited positive frequency shifts. Additionally, the correlation function between the two electrodes, $\Delta F^{W E}=f\left(\Delta F^{R E F}\right)$, deviated from the linearity that was obtained from ammonia-free water vapor measurements and appeared below the original calibration curve [Fig. 6(a)]. This result is attributed to the adsorption of ammonia onto the PAA-infused film being faster than the desorption of water molecules. Thus, the frequency of the PAA- 


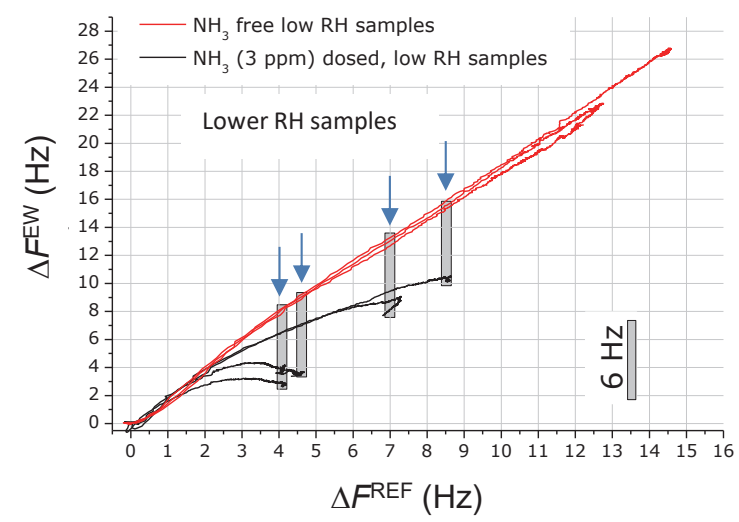

(a)

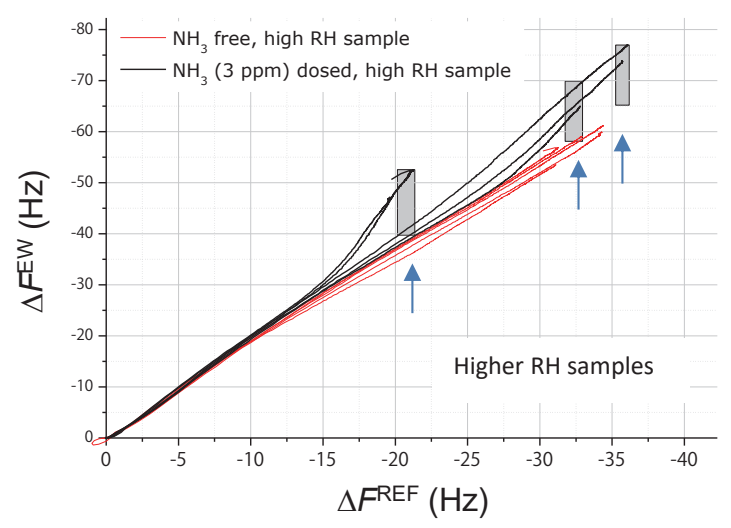

(b)

Fig. 6. (Color online) Behaviors of function $\Delta F^{W E}=f\left(\Delta F^{R E F}\right.$ ) at $\mathrm{RH}$ (a) lower and (b) higher than the humidity level of the baseline. The gray bars in both graphs indicate the values of $\Delta F^{d e v}$.

infused QCM sensor increased more slowly than that of the reference electrode. As previously described, the influence of ammonia was clear in the case of the $\left(\mathrm{SiO}_{2} / \mathrm{PAH}\right)_{10}+\mathrm{PAA}$ film, whereas no effect was observed for the film without PAA. As evident from four independent measurement results shown in Fig. 6(a), even when each response differed (due to small variations in $\mathrm{RH}$ ), the difference between the $\Delta F^{W E}$ values for the ammonia-free samples and the samples dosed with $3 \mathrm{ppm}$ ammonia gas, which is defined as $\Delta F^{d e v}$, was almost the same (approximately $6 \mathrm{~Hz}$, represented as gray bars on the graph).

We added $7 \mu \mathrm{L}$ of aqueous $0.1 \mathrm{wt} \%$ ammonia to a $3 \mathrm{~L}$ sampling bag filled with humidified $\mathrm{N}_{2}$ gas to prepare a highly humidified sample containing $3 \mathrm{ppm}$ gaseous ammonia. In the case of samples dosed with 3 ppm ammonia gas with RH higher than the humidity level of the baseline, departures from the linearity (as indicators of ammonia presence) were still observed but in the opposite direction to the low-RH samples: both water and ammonia were adsorbed, resulting in larger negative frequency shifts at high humidity levels [Fig. 6(b)], with similar values of $\Delta F^{d e v}$. However, the repeatability of the response appeared to decrease according to the increase in humidity. This diminished repeatability is attributable to the competitive adsorption of ammonia and water molecules on a limited number of binding sites in the working electrode; nevertheless, the presence of ammonia was clearly identified.

To determine the ammonia concentration in human breath, we first examined the reference sample that consisted of well-humidified air and represented exhaled human breath with $0 \mathrm{ppm}$ ammonia; ideally, it should be $95 \% \mathrm{RH}$ at room temperature. The first run with this highly humidified air was used for the "calibration run", which was subsequently used to build the function $\Delta F^{W E}=f\left(\Delta F^{R E F}\right)$ and predict the response of the PAA-infused (working) electrode to humidity in ammonia-mixed samples (i.e., human breath, which comprises a mixture of approximately $1 \mathrm{ppm}$ ammonia and $95 \% \mathrm{RH}$ ). Finally, the obtained $\Delta F^{d e v}$ was treated as a response to the ammonia gas present in human breath. Preliminary tests with $3 \mathrm{ppm}$ doses of ammonia in breath were conducted; the resulting data obtained from the $\left(\mathrm{SiO}_{2} / \mathrm{PAH}\right)_{10}+\mathrm{PAA}$ working electrode are summarized in Fig. 7(a), where they are also compared with the data for samples with the same ammonia concentration in different matrices. 


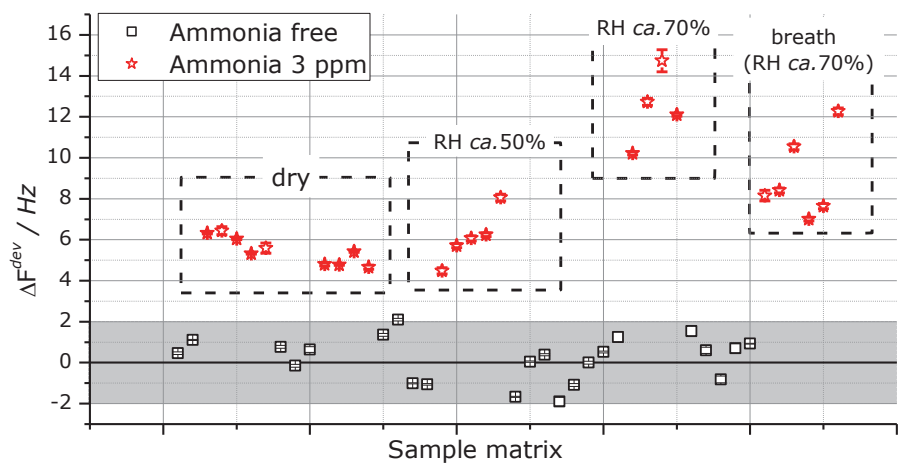

(a)

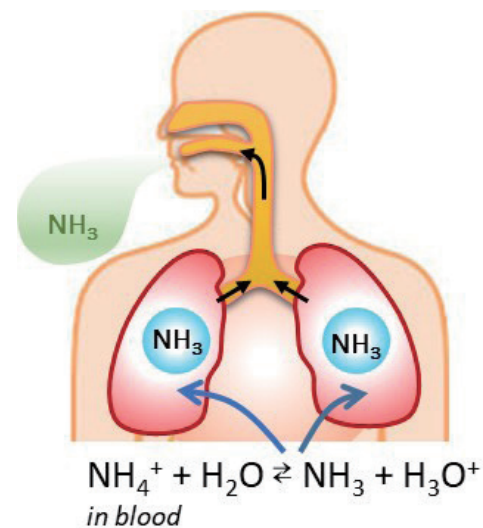

(b)

Fig. 7. (Color online) (a) Sensor responses obtained using a two-QCM electrode system for samples dosed with $3 \mathrm{ppm}$ ammonia gas in different matrices (dry, ca. 50\% RH, ca. 70\% RH, and breath), where the RE and WE were modified with $\left(\mathrm{SiO}_{2} / \mathrm{PAH}\right)_{10}$ and $\left(\mathrm{SiO}_{2} / \mathrm{PAH}\right)_{10}+\mathrm{PAA}$ films, respectively.

Obviously, these results confirm that $3 \mathrm{ppm}$ ammonia detection in the different matrices can be realized. Both the initial investigations into diagnostic breath-ammonia monitoring and the current status of technology indicate that breath analysis has great potential as a noninvasive means of diagnosis. ${ }^{(2)}$ As schematically shown in Fig. 7(b), ammonia is an important disease biomarker associated with renal insufficiency and is emitted in human breath. The ideal breath ammonia monitoring device, as described by Hibbard and Killard, ${ }^{(42)}$ would be one that is specifically sensitive to ammonia gas and capable of detecting it at physiologically relevant concentrations in the ppb range with: (1) good precision and accuracy, (2) insensitivity to interferences, (3) portability for point-of-care use, (4) better ease-of-use for the user, (5) realtime display of measurements, and (6) low cost.

As recently mentioned by Dai et al. ${ }^{(34)}$ breath ammonia can also be a powerful biomarker of acute hepatic injury as well as other human health disorders. ${ }^{(43)}$ Evidently, our current sensor system that comprises only two QCM sensors provides a novel measurement protocol for ammonia gas detection under real humidity conditions.

\section{Conclusions}

QCM-based environmental sensors are rare owing to their disadvantages. In particular, the high sensitivity of QCM-based devices to humidity limits their practical application as gas-sensing devices. To solve this problem, differentiation of QCM output signals was demonstrated, which enabled us to distinguish environmental changes related to humidity, smoke, or gas exposure. Systems based on surface-modified QCMs can thus be implemented as alarms for volatile-organic-compound-related threats, or as triggers for automatic ventilation in buildings with toxic compound saturation risks. Interestingly, a newly developed two-QCM electrode sensor system that comprises only two QCM sensors provided a novel measurement protocol for ammonia gas detection under real humidity conditions. This humidity influence 
extraction approach enabled ammonia detection in cases where parallel humidity changes occurred. LODs approaching $2 \mathrm{ppm}$ aqueous ammonia (equivalent to ca. $0.5 \mathrm{ppm}$ gaseous) were achieved, which allow breath ammonia at pathological levels (greater than $3 \mathrm{ppm}$ ) to be measured. For future work, we expect that the current sensor system will be used for multipurpose assessments of human breath and respiratory patterns.

\section{Acknowledgments}

S.-W. Lee acknowledges a Grant-in-Aid for Scientific Research (A) (15H01713) from the Japan Society for the Promotion of Science.

\section{References}

1 T. C. Pearce, S. S. Schiffman, H. T. Nagle, and J. W. Gardner: Handbook of Machine Olfaction: Electronic Nose Technology (Wiley-VCH Verlag GmbH \& Co. KGaA, Weinheim, 2003).

2 F. Röck, N. Barsan, and U. Weimar: Chem. Rev. 108 (2008) 705.

3 A. Jinks and D. G. Laing: Physiol. Behav. 72 (2001) 51.

4 G. Peng, U. Tisch, O. Adams, M. Hakim, N. Shehada, Y. Y. Broza, S. Billan, R. Abdah-Bortnyak, A. Kuten, and H. Haick: Nat. Nanotechnol. 4 (2009) 669.

5 F. Sauerbrey: Zeit Physik 155 (1959) 206 (in German).

6 B. Beckera and M. A. Cooper: J. Mol. Recognit. 24 (2011) 754.

7 R. E. Speight and M. A. Cooper: J. Mol. Recognit. 25 (2012) 451.

8 A. Michanek, N. Kristen, F. Höök, T. Nylander, and E. Sparr: Biochim. Biophys. Acta Biomembr. 1798 (2010) 829.

9 A. R. Tehrani-Bagha and K. Holmberg: Langmuir 24 (2008) 6140.

10 N. J. Cho, C. W. Frank, B. Kasemo, and F. Höök: Nat. Protoc. 5 (2010) 1096.

11 R. Hao, D. Wang, X. Zhang, G. Zuo, H. Wei, R. Yang, Z. Zhang, Z. Cheng, Y. Guo, and Z. Cui: Biosens. Bioelectron. 24 (2009) 1330.

12 N. Mizutani, S. Korposh, R. Selyanchyn, S. Wakamatsu, and S. W. Lee: Sens. Transducers 137 (2012) 1.

13 R. L. Caygill, G. E. Blair, and P. A. Millner: Anal. Chim. Acta 681 (2010) 8.

14 Q. Chen, W. Tang, D. Wang, X. Wu, N. Li, and F. Liu: Biosens. Bioelectron. 26 (2010) 575.

15 A. Pietrzyk, S. Suriyanarayanan, W. Kutner, R. Chitta, M. E. Zandler, and F. D'Souza: Biosens. Bioelectron. 25 (2010) 2522.

16 H. Huang, J. Zhou, S. Chen, L. Zeng, and Y. Huang: Sens. Actuators, B 101 (2004) 316.

17 S. W. Lee, N. Takahara, S. Korposh, D. H. Yang, K. Toko, and T. Kunitake: Anal. Chem. 82 (2010) 2228.

18 Z. Pei, X. Ma, P. Ding, W. Zhang, Z. Luo, and G. Li: Sensors 10 (2010) 8275.

19 E. A. Baldwin, J. Bai, A. Plotto, and S. Dea: Sensors 11 (2011) 4744.

20 C. I. L. Justino, T. A. Rocha-Santos, and A. C. Duarte: TrAC, Trends Anal. Chem. 29 (2010) 1117.

21 S. Korposh, R. Selyanchyn, and S. W. Lee: Sens. Actuators, B 147 (2010) 599.

22 R. Selyanchyn, S. Korposh, S. Wakamatsu, and S. W. Lee: Anal. Sci. 27 (2011) 253.

23 R. Selyanchyn, S. Korposh, S. Wakamatsu, and S. W. Lee: Sensors 11 (2011) 1177.

24 R. Selyanchyn, S. Wakamatsu, K. Hayashi, and S. W. Lee: Sensors 15 (2015) 18834.

25 Y. Ogimoto, R. Selyanchyn, N. Takahara, S. Wakamatsu, and S. W. Lee: Sens. Actuators, B 215 (2015) 428.

26 S. Herberger, M. Herold, H. Ulmer, A. Burdack-Freitag, and F. Mayer: Build. Environ. 45 (2010) 2430.

27 A. Cowlard, W. Jahn, C. Abecassis-Empis, G. Rein, and J. Torero: Fire Technol. 46 (2008) 719.

28 W. R. Fahrner, R. Job, and M. Werner: Microsyst. Technol. 7 (2000) 138.

29 K. Koistinen, D. Kotzias, S. Kephalopoulos, C. Schlitt, P. Carrer, M. Jantunen, S. Kirchner, J. McLaughlin, L. Mølhave, E. O. Fernandes, and B. Seifert: Allergy 63 (2008) 810.

30 M. Intorp, S. Purkis, M. Whittaker, and W. Wright: Contrib. Tob. Res. 23 (2009) 161.

31 J. M. Ribó, J. Crusats, J.-A. Farrera, and M. L. Valero: J. Chem. Soc., Chem. Commun. 6 (1994) 681.

32 T. Wang, W. Yasukochi, S. Korposh, S. W. James, R. P. Tatam, and S. W. Lee: Sens. Actuators, B 228 (2016) 573.

33 B. Timmer, W. Olthuis, and A. Van Den Berg: Sens. Actuators, B 107 (2005) 666. 
34 M. Z. Dai, Y. L. Lin, H. C. Lin, H. W. Zan, K. T. Chang, H. F. Meng, J. W. Liao, M. J. Tsai, and H. Cheng: Anal. Chem. 85 (2013) 3110.

35 S. Davies, P. Spanel, and D. Smith: Kidney Int. 52 (1997) 223.

36 J. P. Ong, A. Aggarwal, D. Krieger, K. A. Easley, M. T. Karafa, F. Van Lente, A. C. Arroliga, and K. D. Mullen: Am. J. Med. 114 (2003) 188.

37 D. J. Kearney, T. Hubbard, and D. Putnam: Digest. Dis. Sci. 47 (2002) 2523.

38 A. Amano, Y. Yoshida, T. Oho, and T. Koga: Oral Surg. Oral Med. Oral Pathol. Oral Radiol. Endod. 94 (2002) 692.

39 M. Bendahan, P. Lauque, C. Lambert-Mauriat, H. Carchano, and J. L. Seguin: Sens. Actuators, B 84 (2002) 6.

40 B. Ding, M. Yamazaki, and S. Shiratori: Sens. Actuators, B 106 (2005) 477.

41 M. Hoerter, A. Oprea, N. Bârsan, and U. Weimar: Sens. Actuators, B 134 (2008) 743.

42 T. Hibbard and A. J. Killard: Crit. Rev. Anal. Chem. 41 (2011) 21.

43 D. Smith and P. Španěl: Analyst 132 (2007) 390.

\section{About the Authors}

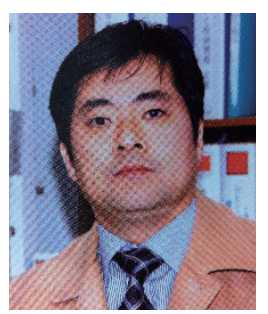

Seung-Woo Lee obtained his Ph.D. degree in chemistry and biochemistry from Kyushu University, Japan, in 1999. He is a professor in the Faculty of Environmental Engineering at the University of Kitakyushu, Japan. His current research interests include the self-assembly and functionalization of organic/inorganic nanohybrids and nanoparticles, molecular imprinting using metal oxide or hybrid thin films, and chemical sensors for biomedical applications.

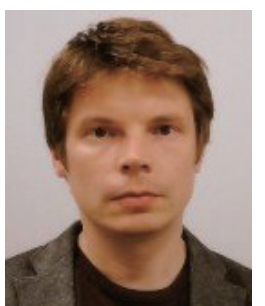

Roman Selyanchyn obtained his Ph.D. degree from the University of Kitakyushu, Japan, in 2012 and is currently a postdoctoral researcher in the International Institute for Carbon Neutral Energy Research (I2CNER), Kyushu University, Japan. His research interests include biomedical applications of analytical instruments and chemical sensors. In particular, he is interested in the applications of thin films for sensing and as a part of gas separation membranes (for $\mathrm{CO}_{2}$ capture technology).

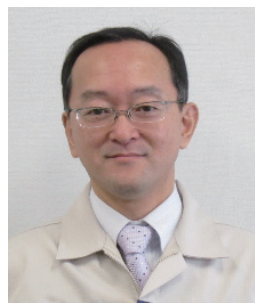

Shunichi Wakamatsu obtained his bachelor degree from the University of Tohoku Institute of Technology, Japan, in 1986. He joined Hitachi Kokusai Electric Inc. in the same year. $\mathrm{He}$ is currently a General Manager at the Chitose Technical Center of Nihon Dempa Kogyo Co., Ltd. His research interests focus on quartz-crystal-device sensor systems.

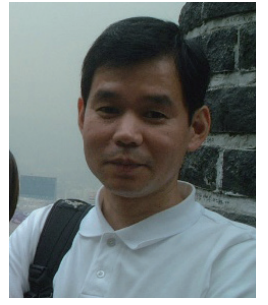

Kenshi Hayashi received his B.E., M.E., and Ph.D. degrees, all in electrical engineering, from Kyushu University, Japan, in 1982, 1984, and 1988, respectively. He is a professor at the Graduate School of Information Science and Electrical Engineering of Kyushu University. He is a member of the Japan Society of Applied Physics and the Institute of Electrical Engineers of Japan. His current research interests include odor sensing and evaluation, the visualization of odor space, nanowire sensors, and plasmonic sensors. 